\title{
EchoGéo
}

$10 \mid 2009$

La piraterie

\section{La géographie en classe préparatoire ESC voie scientifique}

\section{Élizabeth Crémieu}

\section{(2) OpenEdition}

12 Journals

\section{Édition électronique}

URL : https://journals.openedition.org/echogeo/11463

DOI : $10.4000 /$ echogeo. 11463

ISSN : 1963-1197

\section{Éditeur}

Pôle de recherche pour l'organisation et la diffusion de l'information géographique (CNRS UMR 8586)

Référence électronique

Élizabeth Crémieu, « La géographie en classe préparatoire ESC voie scientifique », EchoGéo [En ligne], 10 | 2009, mis en ligne le 06 novembre 2009, consulté le 16 septembre 2021. URL : http:// journals.openedition.org/echogeo/11463; DOI : https://doi.org/10.4000/echogeo.11463

Ce document a été généré automatiquement le 16 septembre 2021.

EchoGéo est mis à disposition selon les termes de la licence Creative Commons Attribution - Pas d'Utilisation Commerciale - Pas de Modification 4.0 International (CC BY-NC-ND) 


\title{
La géographie en classe préparatoire ESC voie scientifique
}

\author{
Élizabeth Crémieu
}

1 Les élèves de classe préparatoire ESC voie scientifique suivent un enseignement d'Histoire, géographie et géopolitique du monde contemporain (HGGMC), à raison de six heures par semaine pendant deux ans. Objectif de cet enseignement : donner aux élèves des outils intellectuels et des connaissances leur permettant de comprendre le monde, ses aspects politiques, géopolitiques, économiques, sociaux, culturels,en s'appuyant sur l'histoire et la géographie. On peut d'ailleurs remarquer que depuis quelques années, beaucoup d'écoles de commerce prolongent l'enseignement d'HGG des classes préparatoires par des cours de géopolitique. L'HGG est importante par les coefficients aux concours : coefficient 6 à HEC sur 30 coefficients à l'écrit, coefficient 5 dans la plupart des écoles (toujours sur 30 coefficients à l'écrit).

La géographie est donc enseignée en même temps que l'histoire et la géopolitique, par le même enseignant, qui n'est pas nécessairement géographe, et qui est même le plus souvent historien.

3 Le programme a été renouvelé il y a 4 ans par une équipe où les professeurs de classe préparatoire étaient représentés, pour y introduire la géopolitique. Il est découpé en 4 modules semestriels:

- en première année, le module 1 retrace l'évolution du monde du début du XX $\mathrm{X}^{\mathrm{e}}$ siècle à la rupture des années 1970. Il s'agit de géopolitique et d'histoire économique, la géographie est ici quasi absente. Le module 2 porte sur la mondialisation, d'abord en montrant sa mise en place, puis en étudiant ses acteurs, ses territoires, ses enjeux .Ce module est donc beaucoup plus géographique que le premier: ainsi le thème des frontières peut-il être traité à ce moment-là, avant d'être repris et développé l'année suivante ;

- en deuxième année (modules 3 et 4), la géographie devient centrale puisqu'il s'agit d'étudier la géodynamique des grands ensembles continentaux : Amériques, Asie, Europe, Afrique et Moyen-Orient. La géographie physique, sans être totalement absente, est réduite, le programme privilégie la géographie économique ainsi que la géopolitique, sans négliger les aspects culturels, qui conduisent à rappeler les traits essentiels des grandes civilisations. 
4 heures, a partir dun sujet accompagné de 2 a 4 pages de documents (statistiques, 4 heures, à partir d'un sujet accompagné de 2 à 4 pages de documents (statistiques, courbes, cartes). Les sujets sont des sujets de synthèse, souvent ambitieux. Tous les candidats travaillent, les connaissances essentielles leur sont fournies par les documents, il s'agit donc de distinguer ceux qui savent le mieux réfléchir, ce qui est à vrai dire l'ambition de toutes les épreuves de tous les concours!

$5 \mathrm{Au}$ concours commun HEC-ESCP, les élèves doivent en plus de la dissertation réaliser une carte, le fond de carte et le sujet étant fournis, et ceci toujours en 4 heures. Même exigence à un sujet sur deux du concours Ecricome, commun à plusieurs écoles de province. Seule originalité : l'épreuve spécifique du concours ESC, qui se décompose en une dissertation, une question de cours, un croquis. Disons tout de suite que donner quatre heures aux élèves pour une dissertation et une carte est une exigence très élevée, trop élevée (opinion personnelle de l'auteur du présent article, qui a demandé sans succès à plusieurs reprises que l'épreuve soit allongée d'une demi-heure). Le président du jury de l'ESSEC, ancien professeur de classe préparatoire au lycée Henri $\mathrm{IV}$, ne pense pas autrement comme il l'a dit dans une interview à Alternatives economiques.

6 En ce qui concerne la carte, le concours Ecricome va innover l'an prochain : les élèves ne devront plus réaliser une carte, mais commenter une carte géopolitique. Ce qui est intéressant, intelligent, mais n'allège pas l'épreuve!

7 Donnons en exemples les sujets posés aux concours en 2009:

- ESSEC : L'essor économique et la montée en puissance de la Chine : chances ou menaces pour le reste du monde? Sujet accompagné d'une chronologie et d'une carte montrant la chine et ses voisins ;

- sujet commun ESCP-EAP-HEC : les Amériques entre intégrations et fragmentations. Sujet accompagné d'une chronologie, d'une carte du Mexique : richesses naturelles, minorités et opposition armée, d'une carte de la Colombie : trafics et mouvements armés, et de 3 documents : les PNB du continent américain, l'évolution de la balance commerciale du Mexique de 1994 à 2004 et les exportations et importations du Mercosur en 2003,4,5. La dissertation doit obligatoirement être complétée par la réalisation d'une carte (fond de carte fourni et sujet imposé) ;

- sujet Ecricome $n^{\circ} 1:$ les dynamiques démographiques et leurs incidences économiques, sociales et géopolitiques dans le monde d'aujourd'hui. Le sujet est accompagné de 4 documents d'appui. Avec un croquis obligatoire : les migrations internationales de population au début du XXI ${ }^{e}$ siècle ;

- sujet Ecricome $n^{\circ} 2$ : les Etats-Unis d'Amérique : la fin de ler « Empire »?

- Enfin il y a de l'HGGMC à l'oral de la plus prestigieuse des écoles, HEC, avec un gros coefficient. Tandis que l'ESC Grenoble a introduit la géopolitique en option à l'oral : les candidats peuvent commenter une carte géopolitique en introduction à l'entretien de personnalité.

8 Avec une telle feuille de route (expression très à la mode) comment travaille-t-on en classe préparatoire ESC, sachant que les rédacteurs du programme actuel souhaitaient que de nouvelles méthodes de travail soient développées? Partout le cours magistral est privilégié, pour des raisons de temps. Les élèves en plus du cours mettent en fiches une partie des manuels. Et ils apprennent à réaliser des cartes. Le temps manque pour des exposés (lourds à préparer pour ceux qui les font, pas très convaincants pour ceux qui les écoutent et qui préfèrent la parole du professeur), pour faire réaliser des

EchoGéo, 10 | 2009 
travaux personnels, pour faire des recherches. Il faut à la fois de la profondeur historique (en $2^{\mathrm{e}}$ année, on étudie Japon, Chine, Inde) et coller à l'actualité.

De nombreux manuels ont été rédigés pour les élèves de prépa ESC (qui représentent un marché "de niche » mais non négligeable) : les uns par des professeurs de classe préparatoire (aux PUF) les autres par des équipes associant universitaires et professeurs de classe préparatoire (chez Nathan et Bréal). Les élèves doivent aussi connaître (et donc lire) les géographes contemporains : citons Bernard Bret, Sylvie Brunel, Michel Foucher, Thierry Sanjuan... Enfin ils sont invités à lire des revues, des essais, des textes littéraires : Ebène, de Kapuscinski, est une extraordinaire introduction à l'Afrique, très accessible pour des élèves surchargés de travail ; Voyages aux pays $d u$ coton, d'Orsenna, est un must. Les enseignants se tiennent au courant des avancées de la recherche en lisant les revues de géographie, en utilisant Géoconfluences, en allant sur internet, et les utilisent pour nourrir et actualiser leurs cours.

10 Les classes préparatoires ESC ont intégré certains outils informatiques : avec une clé USB, de nombreuses cartes et documents sont disponibles en salle de classe avec la possibilité de les envoyer ensuite par courriel aux élèves qui les étudient à leur guise. Pour les cartes, les enseignants puisent dans la cartothèque de Sciences Po et dans Le monde Diplomatique. Notons aussi le recours à l'émission de Jean Christophe Victor, Le Dessous des Cartes

11 La classe préparatoire ESC est donc une des filières où on fait beaucoup de géographie économique, et de géopolitique. Les élèves apprécient souvent ce cours, en prise sur le monde actuel, et consentent un énorme travail pendant deux ans pour acquérir méthodes et connaissances.

12 Il y a des écueils à éviter : le programme est tellement vaste que l'on court toujours le danger du survol, de la superficialité. Ceci d'autant plus que les changements de programme et l'allègement des horaires dans le secondaire nous amènent des élèves de moins en moins formés : en deux ans, il faut passer de définitions de base (citons par exemple : agriculture extensive et intensive, aménagement du territoire, ville globale...) à des sujets pointus comme ceux qui sont posés aux concours (voir supra)

13 Nous avons demandé au professeur d'une classe préparatoire prestigieuse de donner son avis.

\section{Interview dans la revue Géoconfluences}

\section{4 - Quelle définition donneriez-vous de la géographie dans les programmes?}

15 L'originalité du programme des classes préparatoires économiques et commerciales tient à l'association entre histoire, géographie et géopolitique, sans négliger une forte dose d'économie. C'est sa richesse. La géographie que nous pratiquons est donc à la fois une géographie historique, une géographie économique et une géographie politique. Je pourrais aussi bien dire qu'il s'agit d'une histoire géographique, économique et géopolitique. Le terme le meilleur que j'ai trouvé pour désigner notre champ d'étude est la géoéconomie - mais on ne va pas encore ajouter une discipline à toutes celles que nous devons enseigner, et du coup étudier...!

- Quelle place accordez-vous à la géographie dans votre enseignement? 
17 Tout et rien, puisque je m'efforce de ne jamais oublier les quatre dimensions de notre programme. Pas un cours où la géographie ne soit présente, pas un cours qu'elle occupe totalement.

Le but qui nous est assigné, me semble-t-il, est d'expliquer les rapports de force du monde actuel - c'est ce que nous impose la géopolitique pour cela nous mobilisons les héritages, les territoires et les intérêts - l'histoire, la géographie et l'économie. qui avait fini par construire un univers totalement abstrait, coupé de la réalité ?

\section{AUTEUR \\ ÉLIZABETH CRÉMIEU}

Elizabeth Crémieu (elizabethcremieu@orange.fr) est professeur en classes préparatoires. 